\title{
Microwave Versus Radiofrequency Ablation for the Treatment of Liver Malignancies: A Randomized Controlled Phase 2 Trial
}

\author{
Aleksandar Radosevic ( $\square$ aradosevic@psmar.cat ) \\ Department of Radiology, Hospital del Mar, Barcelona
}

Rita Quesada

Department of Experimental and Health Sciences, Universitat Pompeu Fabra, Barcelona

\section{Clara Serlavos}

Department of Radiology, Hospital del Mar, Barcelona

\section{Juan Sánchez}

Department of Radiology, Hospital del Mar, Barcelona

\section{Ander Zugazaga}

Department of Radiology, Hospital del Mar, Barcelona

\section{Ana Sierra}

Department of Radiology, Hospital del Mar, Barcelona

\section{Susana Coll}

Department of Hepatology, Hospital del Mar, Barcelona

\section{Marcos Busto}

Department of Radiology, Hospital del Mar, Barcelona

\section{Guadalupe Aguilar}

Department of Radiology, Hospital del Mar, Barcelona

\section{Daniel Flores}

Department of Radiology, Hospital del Mar, Barcelona Javier Arce

Department of Radiology, Hospital del Mar, Barcelona José María Maiques

Department of Radiology, Hospital del Mar, Barcelona

\section{Montserrat García}

Department of Hepatology, Hospital del Mar, Barcelona José Antonio Carrion

Department of Hepatology, Hospital del Mar, Barcelona

\section{Laura Visa}

Department of Oncology-IMIM-Ciberonc, Hospital del Mar, Barcelona

\section{María Villamonte}


Department of Surgery, Hospital del Mar, Barcelona

\section{Eva Pueyo}

Department of Surgery, Hospital del Mar, Barcelona

\section{Enrique Berjano}

BioMIT, Department of Electronic Engineering, Universitat Politècnica de València, Valencia

\section{Macarena Trujillo}

BioMIT, Department of Applied Mathematics, Universitat Politècnica de València, Valencia

\section{Patricia Sánchez-Velázquez}

Department of Surgery, Hospital del Mar, Barcelona

\section{Luís Grande}

Department of Surgery, Hospital del Mar, Barcelona

\section{Fernando Burdio}

Department of Surgery, Hospital del Mar, Barcelona

\section{Research Article}

Keywords: Hepatocellular carcinoma, liver metastases, liver tumors, radiofrequency ablation, microwave ablation

Posted Date: May 25th, 2021

DOI: https://doi.org/10.21203/rs.3.rs-508761/v1

License: (c) (1) This work is licensed under a Creative Commons Attribution 4.0 International License. Read Full License

Version of Record: A version of this preprint was published at Scientific Reports on January 10th, 2022. See the published version at https://doi.org/10.1038/s41598-021-03802-x. 


\section{Abstract}

Purpose: Microwave (MWA) and radiofrequency ablation (RFA) are main ablative techniques for hepatocellular carcinoma (HCC) and colorectal liver metastasis (MT). This randomized phase 2 clinical trial compares the effectiveness of MWA and RFA in medium-sized liver tumors.

Methods: HCC and MT patients with 1.5 to $4 \mathrm{~cm}$ tumors suitable for ablation were randomized into MWA or RFA Groups. The primary endpoints were primary technical success (TS) and local tumor progression (LTP) rate after a 2-year follow-up. Secondary endpoints were safety and overall survival.

Results: Between June 2015 and April 2020, 82 patients were randomly assigned (41 patients per group). For the per-protocol analysis, three patients were excluded. Median follow-up was 27 months (MWA group) and 23 months (RFA Group). The TS was achieved in 98\% (46/47) and $90 \%(45 / 50)(p=0.108)$, and LTP was observed in $21 \%(10 / 47)$ vs. $12 \%(6 / 50)$ (OR 1.9 [95\% Cl 0.66-5.3], p=0.238) of tumors in the MWA and RFA Group, respectively. Major complications were found in 5 cases $(11 \%)$ in the MWA Group vs. 2 cases (4\%) in RFA, without statistical significance. MWA created larger ablation zones than RFA $(p=0.036)$.

Conclusion: MWA and RFA show similar effectiveness and safety in medium-sized liver tumors (1.5-4 $\mathrm{cm})$.

\section{Introduction}

Ablation is the first-line option for patients with small $(<2 \mathrm{~cm})$ hepatocellular carcinoma (HCC), who are not potential liver transplant candidates, and in patients with up to three $3 \mathrm{~cm}$ or smaller nodules with associated diseases, according to the Barcelona Clinic Liver Cancer group (BCLC) ${ }^{1}$. On an individual basis, ablation can also be considered in patients with larger tumors $(3-5 \mathrm{~cm})$ and advanced liver disease (Child-Pugh score B) ${ }^{1,2}$. Likewise, the European Society for Medical Oncology (ESMO) also includes thermal ablation as part of the treatment algorithm for oligometastatic colorectal disease ${ }^{3}$. Most authors agree that ablation is considered for small $(<3 \mathrm{~cm})$ colorectal liver metastasis (MT) in patients who are unsuitable for resection due to impaired general health, a history of extensive abdominal surgery, lesions in an unfavorable location or insufficient future liver remnant 4 .

Radiofrequency ablation (RFA) is a mature and well-known thermal ablation technique which has been shown to be both safe and effective for treating liver nodules ${ }^{2,5,6}$. However, liver tumors adjacent to large vessels may often be incompletely ablated due to the heat sink effect, which can lead to Local Tumor Progression (LTP) ${ }^{5,7}$. Microwave ablation (MWA) using the most advanced devices obtains shorter ablation times, higher ablation temperatures, larger ablation zones and a weakened heat-sink effect than RFA, all of which are considered to improve the efficacy of tumor ablation ${ }^{8-11}$. However, these theoretical advantages of MWA could also be its flaws because being more effective it can easily injure adjacent critical structures (e.g. nearby bile or portal structures) ${ }^{12}$. This is mainly because MWA power can 
propagate thorough materials with much lower electrical conductivity than RFA, which requires an electrically conductive path, which is interrupted when the electrodes enter desiccated tissue, which inevitably appears after a certain application time $10,11,13,14$. The actual ablation zone created by MWA may thus be larger, but not readily apparent on immediate post-ablation imaging ${ }^{15}$ and may be more elongated or more ellipsoid than RFA (i.e. with a lower Short to Long diameter Ratio-SLR-) ${ }^{16-19}$. In fact, for many authors the major limitation of conventional MWA systems is the fact of being unable to predict ablation zone size and shape ${ }^{5,20}$. However, ablation zone predictability is expected to improve with advanced MWA systems $5,20,21$.

Several recent randomized clinical trials (RCT) have compared different classical RFA devices (e.g. cooltip applicators) with the latest MWA devices used to treat a combination of multiple small and mediumsized tumors. These RCT often obtained similar or slightly better LTP for MWA as the main endpoint in order to draw definitive conclusions ${ }^{19,22-24}$. For example, Vietti Violi et al. ${ }^{24}$ observed $6 \%$ and $12 \%$ LTP at 2 years for MWA and RFA, respectively, even though these differences did not reach statistical significance (median follow-up 26 months). Our aim in the present RCT was therefore to compare the effectiveness of MWA and RFA in medium-sized liver tumors (1.5-4 cm diameter). The MWA procedure was performed by means of an up-to-date technique ( $2.45 \mathrm{GHz}$ generator) and the RFA by means of a hybrid applicator previously shown to be more efficient in the size and shape of the ablation zone than the conventional cool-tip applicator ${ }^{25-27 .}$

\section{Material And Methods \\ 2.1. Study design}

A prospective, randomized, parallel-group, single-blinded phase 2 clinical trial was conducted by the Department of Radiology and Surgery at the Hospital del Mar, a tertiary care hospital in Barcelona, Spain. The study protocol was approved by the Clinical Research and Ethics Committee of the Hospital (June 27th, 2012, Ref 2012/4776/I) and by the Spanish Agency of Medicines and Medical Devices (June 10th, 2013, Ref: 438/13/EC). All patients gave their written informed consent before the procedure. The study was conducted in accordance with principles of the Declaration of Helsinki and Good Clinical Practice guidelines and was officially registered under ISRCTN73194360 on 13/04/2015 on https://www.isrctn.com providing additional study information.

\subsection{Study population}

Between June 2015 and April 2020, all the HCC or MT patients suitable for local ablation were assessed for eligibility after being discussed at the multidisciplinary tumor board for liver malignancies. Inclusion criteria were: age 18 years or older, a diagnosis of HCC or MT suitable for ablation assessed by crosssectional imaging or biopsy (according to BCLC classification ${ }^{1}$ or ESMO guidelines ${ }^{3}$ ), tumor number at presentation $\leq 3$ and the largest tumor diameter between 1.5 to $4 \mathrm{~cm}$. Both percutaneous and surgical approaches were acceptable. The exclusion criteria were: American Anesthesia Association (ASA) 
Classification > III, cardiac pacemaker, thrombocytopenia $<50,000 / \mathrm{mL}$ and previous biliodigestive anastomosis.

\subsection{Randomization, concealment and masking}

The patients who agreed to participate were randomly assigned to either the MWA or RFA Group. Block Randomization ${ }^{28}(1: 1)$ was used to reduce bias and achieve a balance in the allocation of participants to treatment arms. Patient allocation and assignment was by a non-clinician co-author (RQ) not involved in the care of the patients ${ }^{29}$. The patients were masked for the treatment but not the physicians, since different devices were used.

\subsection{Procedures}

A pre-interventional multiphase abdominal CT or MRI scan no older than 30 days (50 days maximum tolerance) was performed and same standard radiological protocol was used on all the patients (basal, arterial, venous and delayed phase scan with 2.5 to $3 \mathrm{~mm}$ slice thickness). All nodules were carefully assessed and recorded for size, segment distribution and unfavorable tumor locations, which were defined as those $5 \mathrm{~mm}$ or closer to critical structures, including the gallbladder, gastrointestinal tract, liver hilum, pericardium, diaphragm, and major vessels (larger than $3 \mathrm{~mm}$ ) ${ }^{30}$.

All the liver tumors included and the targeted ablation zones were semi-automatically delineated on consecutive axial images using Advantage Window Workstation Volume Viewer software (GE Healthcare, Milwaukee, WI, USA) and their volumes were calculated on the portal venous phase acquisition with 2$\mathrm{mm}$ slice reconstruction at the preprocedural and postprocedural (1-month after) CT or MRI (Figure S1), as recommended by Ahmed et al ${ }^{31}$. The digital images were saved and processed on 3D Doctor software (AbleSoftware, Lexington, MA, USA), which extracts information from medical image files to create 3D models. The largest and the two orthogonal diameters were delineated manually using multiplanar reconstruction and $3 \mathrm{D}$ volume rendering similarly to Heering et $\mathrm{al}^{32}$. All the measurements were performed by the same radiologist (AR).

Three radiologists (AR, AZ and JS) with 5-10 years of experience in interventional oncology performed the ablation procedures. The ablation was performed during open and laparoscopic surgery in two patients. In the rest of the patients, ablation was performed percutaneously under conscious sedation with ultrasound guidance in a dedicated ultrasound suite. An artificial ascites or artificial pleural effusion were created in selected cases according to the lesion site. The active ablation time and number of applications of the ablated tumors were recorded.

MWA was performed using a $2.45 \mathrm{GHz}$ MW ablation generator (HS AMICA, HS Hospital Service, Rome, Italy) with a maximum output of $140 \mathrm{~W}$ and a cooled mini-choked 14-gauge antenna. A 3-6 min application at 60-80 W was standard, according to the manufacturer's instructions, as in Amabile et al 33. RFA was conducted with a 200 W generator (CC-3, Radionics, Burlington, MA, USA) using impedance control mode, and a single 14-gauge hybrid internally cooled-perfusion electrode (Gnomon, Apeiron 
Medical, Valencia, Spain) was used following the recommendations described elsewhere for both animals ${ }^{25,27}$ and patients ${ }^{26}$.

Radiological assessment of the ablation procedure was performed 1 month after the intervention, then every 3 months during the first two years and finally every 6 months. In the case of any residual unablated tumor, an additional ablation procedure was performed using the same technique. Primary Technical Success (TS) and LTP were defined as tumor being completely covered by the ablation zone (at 1-month follow-up) and the appearance of any tumor focus in contact with the ablation zone, respectively, as defined by Ahmed et al ${ }^{31}$. Distant hepatic progression was defined as any new hepatic lesion not in contact with the ablation zone. New extrahepatic lesions were considered as metastatic disease. The date of the recurrent disease was determined by the first imaging study showing unequivocal recurrence to ascertain Time to Tumor Progression (TTP).

\subsection{Outcomes}

The primary endpoint was TS (at the one-month control scan) and the proportion of lesions with LTP after 2 years of follow-up. The secondary endpoints were safety and overall survival (OS) ${ }^{31}$. Safety represented all treatment-related complications classified according to the Society of Interventional Radiology guidelines by treated tumor basis taking into account the highest grade ${ }^{34}$. The main study outcomes were also assessment of SLR ${ }^{19}$, sphericity ratio (SR) (the largest diameter to the average of the two remaining diameters of the ablation zone) in which a ratio close to 1 implies greater sphericity ${ }^{35}$, surface area of the ablation zone ${ }^{36}$ and the coefficient of variation (CV) ${ }^{25,35}$ as a measure of reproducibility.

\subsection{Sample size and statistical analysis}

The sample size was based on SLR as the main variable since it was deemed more reliable than survival variables, which are usually difficult to estimate in advance, especially for relatively new treatment techniques. Based on the historic difference in SLR ${ }^{19,25}$ with similar methods of ablation, it was estimated that SLR in the MWA Group would be reduced by $15 \%$. To achieve a power of $80 \%$ in detecting differences with a 2-sided test and a type I error of 0.05 , a total of 82 patients were estimated to be required. All the analyses were performed on the per-protocol population. Since the patients could have multiple lesions, we expected the minimal number of lesions to be greater than the number of patients. Differences in the quantitative data distributions between the two groups were assessed by a two-tailed unpaired Student's $t$ test. Differences in frequencies for categorical data and the proportion of lesions with TS and LTP after 2-year follow-up were assessed by the two-tailed $\chi^{2}$ test. The Odds-Ratio (OR) was calculated with 95\% Confidence Intervals (CI). OS, TTP and LTP were assessed through Kaplan Meier curves using the Log-rank test. Univariate regression with Cox analysis was also calculated to compare time to progression between the two groups. A p-value less than 0.05 was considered to be significant.

\section{Results}


Between June 9, 2015 and April 30, 2020, 145 patients were considered for the trial and 63 (44\%) were excluded for not meeting the inclusion criteria or other reasons (Fig. 1). The remaining 82 patients were randomized to MWA Group $(n=41)$ or RFA Group $(n=41)$. Three patients $(4 \%)$ partially received the allocated intervention, and two (2\%) additional patients were excluded from the analysis due to other diagnoses (intrahepatic cholangiocarcinoma). The final study population was thus 77 patients with 136 tumors (97 larger than $1.5 \mathrm{~cm}$ ) (Fig. 1 and Table 1). As shown in Table 1, patient demographics, diagnosis, ablated tumors per patient, HCC risk factors among others did not differ between the groups, except for a higher age in MWA Group (mean value: 75 vs. 67 years, $p=0.003$ ). 
Table 1

Patient data.

\begin{tabular}{|c|c|c|c|}
\hline & MWA $(n=39)$ & RFA $(n=38)$ & $\mathbf{P}$ \\
\hline Age at inclusion, years & $75(46-93)$ & $67(48-84)$ & 0.003 \\
\hline Men & $22(56 \%)$ & $29(76 \%)$ & 0.065 \\
\hline Diagnosis & $30(77 \%)$ & $28(74 \%)$ & 0.742 \\
\hline Hepatocarcinoma & $9(23 \%)$ & $10(26 \%)$ & \\
\hline \multicolumn{4}{|l|}{ Liver metastases } \\
\hline Number of Ablated Tumors & 67 & 69 & 0.661 \\
\hline Tumors $\geq 1.5 \mathrm{~cm}$ & 47 & 50 & 0.381 \\
\hline Tumors $\geq 3 \mathrm{~cm}$ & 12 & 12 & 0.861 \\
\hline Number of tumors ablated per patient & $22(56 \%)$ & $22(58 \%)$ & 0.468 \\
\hline 1 & $9(23 \%)$ & $8(21 \%)$ & \\
\hline 2 & $7(18 \%)$ & $4(11 \%)$ & \\
\hline 3 & $1(3 \%)$ & $4(11 \%)$ & \\
\hline \multicolumn{4}{|l|}{$>3$} \\
\hline Cirrhosis & $77 \%$ & $71 \%$ & 0.549 \\
\hline BCLC stage & $2(5 \%)$ & $2(5 \%)$ & 0.945 \\
\hline 0 & $28(72 \%)$ & $26(69 \%)$ & \\
\hline$A$ & $9(23 \%)$ & $10(26 \%)$ & \\
\hline \multicolumn{4}{|l|}{ N/A } \\
\hline Child-Pugh Score & $28(72 \%)$ & $21(55 \%)$ & 0.257 \\
\hline A & $2(5 \%)$ & $6(16 \%)$ & \\
\hline B & $9(23 \%)$ & $11(29 \%)$ & \\
\hline \multicolumn{4}{|l|}{$\mathrm{N} / \mathrm{A}$} \\
\hline Previous local treatments* & $10(26 \%)$ & $8(21 \%)$ & 0.634 \\
\hline Concomitant liver surgery & $3(8 \%)$ & $1(3 \%)$ & 0.615 \\
\hline \multicolumn{4}{|l|}{ Data are $n, n(\%)$, or the mean (range). } \\
\hline *Include previous liver surgeries or liv & lations An & s are by pa & \\
\hline
\end{tabular}


There were no differences between the tumor characteristics of both groups, particularly in tumor size and volume (Table 2). However, with similar number of ablations per tumor between both groups, the ablation zones were larger in MWA than in RFA Group $\left(36.8 \mathrm{~cm}^{3} \mathrm{vs} .27 .7 \mathrm{~cm}^{3}, p=0.036\right)$. The shape of the ablation zone was slightly different in the long diameter $(p=0.01)$ but with similar SLR, SR and CV. The surface area seemed to be larger and more variable in MWA Group but it did not reach statistical significance (Table 2). At the 1-month CT, one (2\%) of 47 tumors of medium-sized tumors in MWA Group and five $(10 \%)$ of 50 tumors in RFA Group had signs of residual tumor $(p=0.108)$. All these tumors were treated again with the same technique and all achieved complete response after this additional procedure.

Table 2

Treated tumor information*

\begin{tabular}{|c|c|c|c|}
\hline & $\begin{array}{l}\text { MWA Group } \\
(n=47)\end{array}$ & $\begin{array}{l}\text { RFA Group ( } \mathrm{n}= \\
50)\end{array}$ & p-value \\
\hline Tumor Size (cm) & $2.5(1.5-4.0)$ & $2.4(1.5-4.0)$ & 0.490 \\
\hline Tumor volume $\left(\mathrm{cm}^{3}\right)$ & $5.6(0.6-26.8)$ & $6.3(0.3-28.5)$ & 0.619 \\
\hline Number of ablations per tumor & $2.3(1-6)$ & $2.6(1-7)$ & 0.313 \\
\hline Ablation time (min) per tumor & $8.8(2-25)$ & $11.2(4-32)$ & 0.068 \\
\hline \multicolumn{4}{|l|}{ Size and shape of ablation zone } \\
\hline Long diameter $(\mathrm{cm})$ & $5.83(3.5-9.2)$ & $5.19(3.5-8.2)$ & 0.01 \\
\hline Short diameter $(\mathrm{cm})$ & $2.9(1.7-5.2)$ & $2.8(1.2-5.2)$ & 0.282 \\
\hline Short to long diameter ratio -SLR- & $0.5(0.3-0.7)$ & $0.5(0.3-0.9)$ & 0.229 \\
\hline Volume $\left(\mathrm{cm}^{3}\right)$ & $36.8(9.6-98.3)$ & $27.7(4.9-101)$ & 0.036 \\
\hline Ablation to tumor volume ratio -ATR- & $11.4(2.5-95.0)$ & $10.3(1.1-72.7)$ & 0.672 \\
\hline Sphericity ratio & $1.74(1.2-3.1)$ & $1.69(1.1-2.8)$ & 0.490 \\
\hline Coefficient of variability & $65 \%$ & $64 \%$ & - \\
\hline Surface area $\left(\mathrm{cm}^{2}\right)$ & $\begin{array}{l}70.6(14.9- \\
151.0)\end{array}$ & $62.5(22.9-139.6)$ & 0.173 \\
\hline Unfavorable location $* \star$ & $34(72 \%)$ & $37(74 \%)$ & 0.854 \\
\hline Technical Success (TS) & $46(98 \%)$ & $45(90 \%)$ & 0.108 \\
\hline $\begin{array}{l}\text { Local tumor progression at 2-year follow-up } \\
\text { (LTP) }\end{array}$ & $10(21 \%)$ & $6(12 \%)$ & 0.234 \\
\hline
\end{tabular}


Postprocedural complications are shown in Table 3. Thirteen (27\%) ablated tumors in the MWA and 11 (22\%) in the RFA Group led to complications: two grade 1, fifteen grade 2, four grade 3 and three grade 4 complications were reported, with no significant differences between the groups $(p=0.519)$. No treatment-related deaths were reported.

Table 3

Complications.

\begin{tabular}{|c|c|c|}
\hline & $\begin{array}{l}\text { MWA Group } \\
(n=47)\end{array}$ & $\begin{array}{l}\text { RFA Group } \\
(n=50)\end{array}$ \\
\hline Grade 1 & $0(0 \%)$ & $2(4 \%)$ \\
\hline Asymptomatic biloma & 0 & 1 \\
\hline Peripheral portal vein branch thrombosis & 0 & 1 \\
\hline Grade 2 & $8(17 \%)$ & $7(14 \%)$ \\
\hline Pain requiring additional medication & 4 & 3 \\
\hline Abdominal wall burns & 0 & 1 \\
\hline Postablative syndrome & 4 & 3 \\
\hline Grade 3 & $2(4 \%)$ & $2(4 \%)$ \\
\hline Transitional encephalopathy & 0 & 1 \\
\hline Delayed bilio-bronchial fistula & 1 & 0 \\
\hline Abscess & 1 & 1 \\
\hline Grade 4 & $3(6 \%)$ & $0(0 \%)$ \\
\hline Delayed biliar fistula & 1 & 0 \\
\hline Abscess & 1 & 0 \\
\hline Acute cholecystitis & 1 & 0 \\
\hline Grade 5 & 0 & 0 \\
\hline
\end{tabular}

The median follow-up was similar in both groups (27 months [IQR 19-28] in MWA Group vs. 23 months [IQR 18-28] in RFA Group; $p=0.861$ ). After 2 years, the proportion of tumors with LTP did not differ between groups ( 10 of 47 tumors in MWA Group vs. 6 of 50 in RFA Group, OR = 1.9 [95\% $\mathrm{Cl} 0.66-5.3$ ], $p=$ 0.238). Neither did the Kaplan-Meier analysis of LTP by patient differ between the groups, either in HCC (see Fig. 2A) or in liver metastases ( $p=0.071)$. 
Median TTP was also similar between the MWA and RFA Groups in both diagnoses, in particular 27 months [95\% Cl 19-36] vs. 19 months [95\% Cl 1-36] ( $p=0.329)$, respectively, hazard ratio 1.4 [95\% Cl $0.6-3.1](p=0.413)$, for hepatocarcinoma and 17 months [95\% $\mathrm{Cl} 9-25]$ vs. 6 months $[95 \% \mathrm{Cl} 4-8](p=$ $0.413)$, respectively, hazard ratio $1.1[95 \% \mathrm{Cl} 0.3-3.6](p=0.925)$, for liver metastasis. There were no differences in overall survival between groups in both diagnoses. The 2 -year survival rate was $76 \% \mathrm{vs}$. $81 \%$, respectively, hazard ratio $1.6[95 \% \mathrm{Cl} 0.6-4.3](p=0.378)$, for hepatocarcinoma (see also KaplanMeier analysis in Fig. 2B) and $88 \%$ vs. $56 \%$, respectively, hazard ratio 0.5 [95\% $\mathrm{Cl} 0.9-2.8]$ ( $p=0.440)$, for liver metastasis.

\section{Discussion}

The main finding of this randomized trial was that local control with MWA and RFA is similar for mediumsized liver tumors (1.5 to $4 \mathrm{~cm}$ in diameter) with no difference in oncological outcomes in either group. Although the definition of medium-sized tumors differs among authors ${ }^{31}$, it is clear that local tumor progression in tumors under $2 \mathrm{~cm}$, even with classical RFA devices, is less than $1 \%{ }^{37,38}$, while liver tumors over 4 or $5 \mathrm{~cm}$ may lead to an unacceptable rate of local tumor progression, sometimes over $60 \%$ in classical references ${ }^{37,39}$. In any case, these medium-sized tumors are the everyday challenge of any physician performing thermal ablations, which is why we focused our study on them.

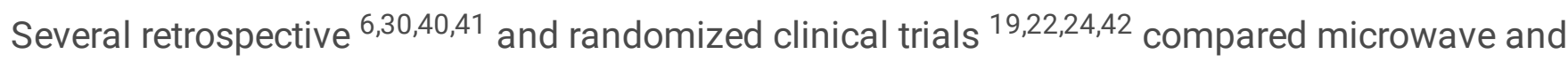
radiofrequency ablation systems dealing with primary or secondary tumors or both. Some of these, however, were carried out with first line thermoablative systems, as in the RCT by Shibata et al ${ }^{42}$, which treated patients with HCC nodules smaller than $4 \mathrm{~cm}$ in diameter and found equivalent results between both techniques with a $24 \%$ overall proportion of local tumor progression in the MWA Group. The RCT conducted by Abdelaziz et a/ ${ }^{22}$ could have been limited by its short follow-up (not specified) and a high lost to follow-up (52\%) ${ }^{43}$. The RCT recently conducted by Vietti Violi et al ${ }^{24}$ compared MWA and RFA in treating HCC in up to three lesions of $4 \mathrm{~cm}$ or less should be highlighted for its well established design. In this last study the authors observed $6 \%$ and $12 \%$ LTP at 2 years for MWA and RFA with no significant differences between groups (median follow-up of 26 months). However, the lesion size in this study was smaller than in our study $(1.8 \mathrm{~cm}$ vs. $2.4 \mathrm{~cm})$ due to the different inclusion criteria. In the present study, after excluding tumors with less than $1.5 \mathrm{~cm}$ with a mean follow-up of 25 months (complete duration of the study: 58 months and no patients lost to follow-up), we observed $21 \%$ and $12 \%$ LTP at 2 years for MWA and RFA, respectively, also with no differences between groups. It also gave an insight into the longterm outcomes not only in $\mathrm{HCC}$ but also in liver metastases with an inherently different natural history.

MWA was initially considered to be less safe than RFA because the ablation zone shape was considered less predictable $17,44,45$. For example, Van Tilborg et al ${ }^{44}$, in a retrospective study with 774 colorectal metastases treated by MWA or RFA in 243 patients, observed that biliary complications (biloma/biliary leakage, biliary obstruction and bilio-pleural fistula) were especially common after peribiliary-MWA (57\%) vs. peribiliary-RFA (3\%) and these significant differences did not decrease with operator experience. 
However, in a multicenter Italian study based on 1037 MWA in 736 patients, major complications were found in only $2.9 \%$ of the patients -similar to historical RFA procedures- ${ }^{20}$. Nor were there any significant differences in device-related complications found in any of the available RCTs comparing MWA and RFA 19,22,24,42. In our study major complications (grade 3-4) were found in 5 cases in the MWA Group (11\%) (including one delayed bilio-bronchial fistula and one delayed biliary fistula) vs. 2 cases in the RFA Group (4\%), with no significant differences between the groups and no procedure-related deaths reported in either group.

Even though both MWA and RFA destroy tissue via thermally coagulative necrosis, they are very different because of their inherently different energy deposition mechanisms, which may explain their many differences in terms of ablation zones ${ }^{10,14}$. Many improvements have been seen in recent years in thermal deposition during ablation, particularly in MWA, which makes it difficult to compare different devices based on the same technology ${ }^{14}$. Even with the latest generation of devices, ablation zone shapes created by MWA tend to be larger, more elongated and maybe less affected by the heat-sink effect $10,16-18,33$, but usually have less control of heat propagation than RFA, possibly because MWA is highly dependent on tissue properties (water content) ${ }^{10,14,15}$. In our study, after a careful 3D reconstruction of each ablation zone, we confirmed that MWA created larger ablation zones mainly because of their more elongated shape (greater long diameter) but were not significantly less predictable taking sphericity ratio, coefficient of variability and surface area into account.

This study had several limitations: first, the trial was performed in a single third referral center with a special dedication to these procedures and a special focus on medium-sized tumors, which are our daily challenge. Although our results are in line with previous RCT results, it is conceivable that some results may differ with different patient inclusion criteria. Second, to reduce the risk of device-related bias we chose to use only one advanced device in each group, even though there are numerous commercially available devices with similar characteristics. In any case, we have to acknowledge that accurate comparisons between MWA and RFA systems are difficult. Third, as in other RCT in this field ${ }^{21,24}$, per protocol analysis was performed to correctly evaluate the true efficacy of each technology (particularly for evaluating ablation zone shape) even though this could be deemed a sub-optimal approach in general terms. Fourth, although a single ablation per tumor is preferable to achieve a reproducible evaluation of ablation zone shape, we performed a mean of more than two ablations per tumor in a pragmatic approach. Although no differences were found between the groups in this last variable and there were no significant differences in coagulation shape among those groups in single ablation per tumor cases (results not shown), some overlapping ablations could mask slight differences in coagulation shape.

\section{Conclusion}

This randomized study showed MWA and RFA obtained similar local tumor progression and technical success in medium-sized liver tumors. After 2-year follow-up we observed no significant difference in the rate of complications and residual unablated tumors, median time to progression or overall survival. 


\section{Declarations}

Acknowledgements: This work was supported by a grant for medical research from Spanish Government (FIS - PI12/00799) and by the Spanish Ministerio de Ciencia, Innovación y Universidades under “Programa Estatal de I+D+i Orientada a los Retos de la Sociedad”, Grant RTI2018-094357-B-C21.

Specific authors contributions: Study design: F.B., R.Q., A.R., L.G., S.C., E.B., M.T., M.G., L.V. and J.C. Literature research: A.R., R.Q., A.S. and F.B. On site procedures: A.R., R.Q., C.S., J.S. and A.Z. Images interpretation and revision: A.R., A.S., M.B., G.A., J.A., D.F., J.S. and A.Z. Data collection: A.R., R.Q., C.S., M.V., A.S. and E.P. Measurements of lesions and coagulation zone volumes: A.R. Figures and tables design: A.R., R.Q. and F.B. Data analysis and data interpretation: A.R., R.Q. and F.B. Manuscript preparation: A.R., R.Q., F.B., E.B., M.T., A.S., L.G. and P.S.V. Access to all data, manuscript revision and approval: All authors.

Competing interest: A.R. and F.B. report non-financial support from HS Hospital Service (Rome, Italy). F.B. is inventor of a patent related with the applicator used in RFA Group (licensed to Apeiron Medical, Valencia, Spain). F.B. also reports personal lectures fees from HS Hospital service and personal lectures fees from Apeiron Medical. The other authors do not have any conflict of interest to disclose.

Data availability statement: Data available on request from the authors.

\section{References}

1. Forner, A., Reig, M. \& Bruix, J. Hepatocellular carcinoma. Lancet (London, England)391, 1301-1314 (2018).

2. Nault, J. C., Sutter, O., Nahon, P., Ganne-Carrié, N. \& Séror, O. Percutaneous treatment of hepatocellular carcinoma: State of the art and innovations. J. Hepatol.68, 783-797 (2018).

3. Van Cutsem, E. et al. ESMO consensus guidelines for the management of patients with metastatic colorectal cancer. Ann. Oncol.27, 1386-1422 (2016).

4. Meijerink, M. R. et al. Radiofrequency and Microwave Ablation Compared to Systemic Chemotherapy and to Partial Hepatectomy in the Treatment of Colorectal Liver Metastases: A Systematic Review and Meta-Analysis. Cardiovasc. Intervent. Radiol.41, 1189-1204 (2018).

5. Sacks, D., McClenny, T. E., Cardella, J. F. \& Lewis, C. A. Society of Interventional Radiology clinical practice guidelines. J. Vasc. Interv. Radiol.14, S199-202 (2003).

6. Ding, J. et al. Comparison of two different thermal techniques for the treatment of hepatocellular carcinoma. Eur. J. Radiol.82, 1379-1384 (2013).

7. Yu, J. et al. A comparison of microwave ablation and bipolar radiofrequency ablation both with an internally cooled probe: Results in ex vivo and in vivo porcine livers. Eur. J. Radiol.79, 124-130 (2011). 
8. Dodd, G. D., Dodd, N. A., Lanctot, A. C. \& Glueck, D. A. Effect of variation of portal venous blood flow on radiofrequency and microwave ablations in a blood-perfused bovine liver model. Radiology267, 129-136 (2013).

9. Primavesi, F. et al. Thermographic real-time-monitoring of surgical radiofrequency and microwave ablation in a perfused porcine liver model. Oncol. Lett.15, 2913-2920 (2018).

10. Brace, C. L. Radiofrequency and Microwave Ablation of the Liver, Lung, Kidney, and Bone: What Are the Differences? Curr. Probl. Diagn. Radiol.38, 135-143 (2009).

11. Rossmann, C., Garrett-Mayer, E., Rattay, F. \& Haemmerich, D. Dynamics of tissue shrinkage during ablative temperature exposures. Physiol. Meas.35, 55-67 (2014).

12. Poulou, L. S., Botsa, E., Thanou, I., Ziakas, P. D. \& Thanos, L. Percutaneous microwave ablation vs radiofrequency ablation in the treatment of hepatocellular carcinoma. World J. Hepatol.7, 10541063 (2015).

13. Amabile, C. et al. Tissue shrinkage in microwave ablation of liver: an ex vivo predictive model. Int. J. Hyperth.33, 101-109 (2017).

14. Kim, C. Understanding the nuances of microwave ablation for more accurate post-treatment assessment. Futur. Oncol.14, 1755-1764 (2018).

15. Lee, J. K. et al. Microwave ablation of liver tumors: degree of tissue contraction as compared to RF ablation. Abdom. Radiol.41, 659-666 (2016).

16. Pillai, K. et al. Heat sink effect on tumor ablation characteristics as observed in monopolar radiofrequency, bipolar radiofrequency, and microwave, using ex vivo calf liver model. Med. (United States)94, e580 (2015).

17. Louis Hinshaw, J., Lubner, M. G., Ziemlewicz, T. J., Lee, F. T. \& Brace, C. L. Percutaneous tumor ablation tools: Microwave, radiofrequency, or cryoablation-what should you use and why? Radiographics34, 1344-1362 (2014).

18. Vogl, T. J. et al. Evaluation of microwave ablation of liver malignancy with enabled constant spatial energy control to achieve a predictable spherical ablation zone. Int. J. Hyperth.34, 492-500 (2018).

19. Di Vece, F., Tombesi, P., Ermili, F., Maraldi, C. \& Sartori, S. Coagulation Areas Produced by Cool-Tip Radiofrequency Ablation and Microwave Ablation Using a Device to Decrease Back-Heating Effects: A Prospective Pilot Study. Cardiovasc. Intervent. Radiol.37, 723-729 (2013).

20. Livraghi, T., Meloni, F., Solbiati, L. \& Zanus, G. Complications of microwave ablation for liver tumors: Results of a multicenter study. Cardiovasc. Intervent. Radiol.35, 868-874 (2012).

21. Kamal, A., Elmoety, A. A. A., Rostom, Y. A. M., Shater, M. S. \& Lashen, S. A. Percutaneous radiofrequency versus microwave ablation for management of hepatocellular carcinoma: $A$ randomized controlled trial. J. Gastrointest. Oncol.10, 562-571 (2019).

22. Abdelaziz, A. et al. Efficacy and survival analysis of percutaneous radiofrequency versus microwave ablation for hepatocellular carcinoma: an egyptian multidisciplinary clinic experience. Surg. Endosc. Other Interv. Tech.28, 3429-3434 (2014). 
23. Yu, J. et al. Percutaneous cooled-probe microwave versus radiofrequency ablation in early-stage hepatocellular carcinoma: A phase III randomised controlled trial. Gut vol. 66 1172-1173 (2017).

24. Violi, N. V. et al. Efficacy of microwave ablation versus radiofrequency ablation for the treatment of hepatocellular carcinoma in patients with chronic liver disease: a randomised controlled phase 2 trial. Lancet Gastroenterol. Hepatol.1253, 1-9 (2018).

25. Burdío, F. et al. Distant infusion of saline may enlarge coagulation volume during radiofrequency ablation of liver tissue using cool-tip electrodes without impairing predictability. Am. J. Roentgenol.196, 837-843 (2011).

26. Ewertowska, E. et al. A clinically oriented computer model for radiofrequency ablation of hepatic tissue with internally cooled wet electrode. Int. J. Hyperth.35, 194-204 (2018).

27. Kho, A. S. K., Foo, J. J., Ooi, E. T. \& Ooi, E. H. Shape-shifting thermal coagulation zone during salineinfused radiofrequency ablation: A computational study on the effects of different infusion location. Comput. Methods Programs Biomed.184, 105289 (2020).

28. Efird, J. Blocked randomization with randomly selected block sizes. Int. J. Environ. Res. Public Health8, 15-20 (2011).

29. Schulz, K. F. \& Grimes, D. A. Allocation concealment in randomised trials: defending against deciphering. Lancet359, 614-618 (2002).

30. Xu, Y. et al. Microwave ablation is as effective as radiofrequency ablation for very-early-stage hepatocellular carcinoma. Chin. J. Cancer36, 14 (2017).

31. Ahmed, M. et al. Image-guided Tumor Ablation: Standardization of Terminology and Reporting Criteria-A 10-Year Update. Radiology273, 241-260 (2014).

32. Heerink, W. J. et al. The relationship between applied energy and ablation zone volume in patients with hepatocellular carcinoma and colorectal liver metastasis. Eur. Radiol.28, 3228-3236 (2018).

33. Amabile, C. et al. Microwave ablation of primary and secondary liver tumours: ex vivo, in vivo, and clinical characterisation. Int. J. Hyperth.33, 34-42 (2017).

34. Khalilzadeh, O. et al. Proposal of a New Adverse Event Classification by the Society of Interventional Radiology Standards of Practice Committee. J. Vasc. Interv. Radiol.28, 1432-1437.e3 (2017).

35. Pereira, P. L. et al. Radiofrequency Ablation: In Vivo Comparison of Four Commercially Available Devices in Pig Livers. Radiology232, 482-490 (2004).

36. Abreu, L. A. dos S. et al. Volume and Shape Assessment of Renal Radiofrequency Ablation Lesion. Urology116, 229.e7-229.e11 (2018).

37. Bruix, J. \& Sherman, M. AASLD PRACTICE GUIDELINE Management of Hepatocellular Carcinoma. 1208-1236 (2005) doi:10.1002/hep.20933.

38. Mulier, S. et al. Local recurrence after hepatic radiofrequency coagulation: Multivariate meta-analysis and review of contributing factors. Annals of Surgery vol. 242 158-171 (2005).

39. Mulier, S. et al. Local recurrence after hepatic radiofrequency coagulation: Multivariate meta-analysis and review of contributing factors. Annals of Surgery vol. 242 158-171 (2005). 
40. Ohmoto, K. et al. Comparison of therapeutic effects between radiofrequency ablation and percutaneous microwave coagulation therapy for small hepatocellular carcinomas. J. Gastroenterol. Hepatol.24, 223-7 (2009).

41. Francica, G. et al. Radiofrequency and microwave ablation of subcapsular hepatocellular carcinoma accessed by direct puncture: Safety and efficacy. Eur. J. Radiol.85, 739-743 (2016).

42. Shibata, T. et al. Small Hepatocellular Carcinoma: Comparison of Radio-frequency Ablation and Percutaneous Microwave Coagulation Therapy. Radiology223, 331-337 (2002).

43. Liao, Y. Y., Ge, D. X. \& Zhang, X. Y. Letter: is microwave ablation superior to radiofrequency ablation for early-stage hepatocellular carcinoma? Aliment. Pharmacol. Ther.48, 1325-1326 (2018).

44. van Tilborg, A. A. J. M. et al. MWA Versus RFA for Perivascular and Peribiliary CRLM: A Retrospective Patient- and Lesion-Based Analysis of Two Historical Cohorts. Cardiovasc. Intervent. Radiol.39, 1438-1446 (2016).

45. Vogl, T. J., Nour-Eldin, N. E. A., Hammerstingl, R. M., Panahi, B. \& Naguib, N. N. N. Microwave Ablation (MWA): Basics, Technique and Results in Primary and Metastatic Liver Neoplasms - Review Article. RoFo Fortschritte auf dem Gebiet der Rontgenstrahlen und der Bildgeb. Verfahren189, 1055-1066 (2017).

\section{Figures}




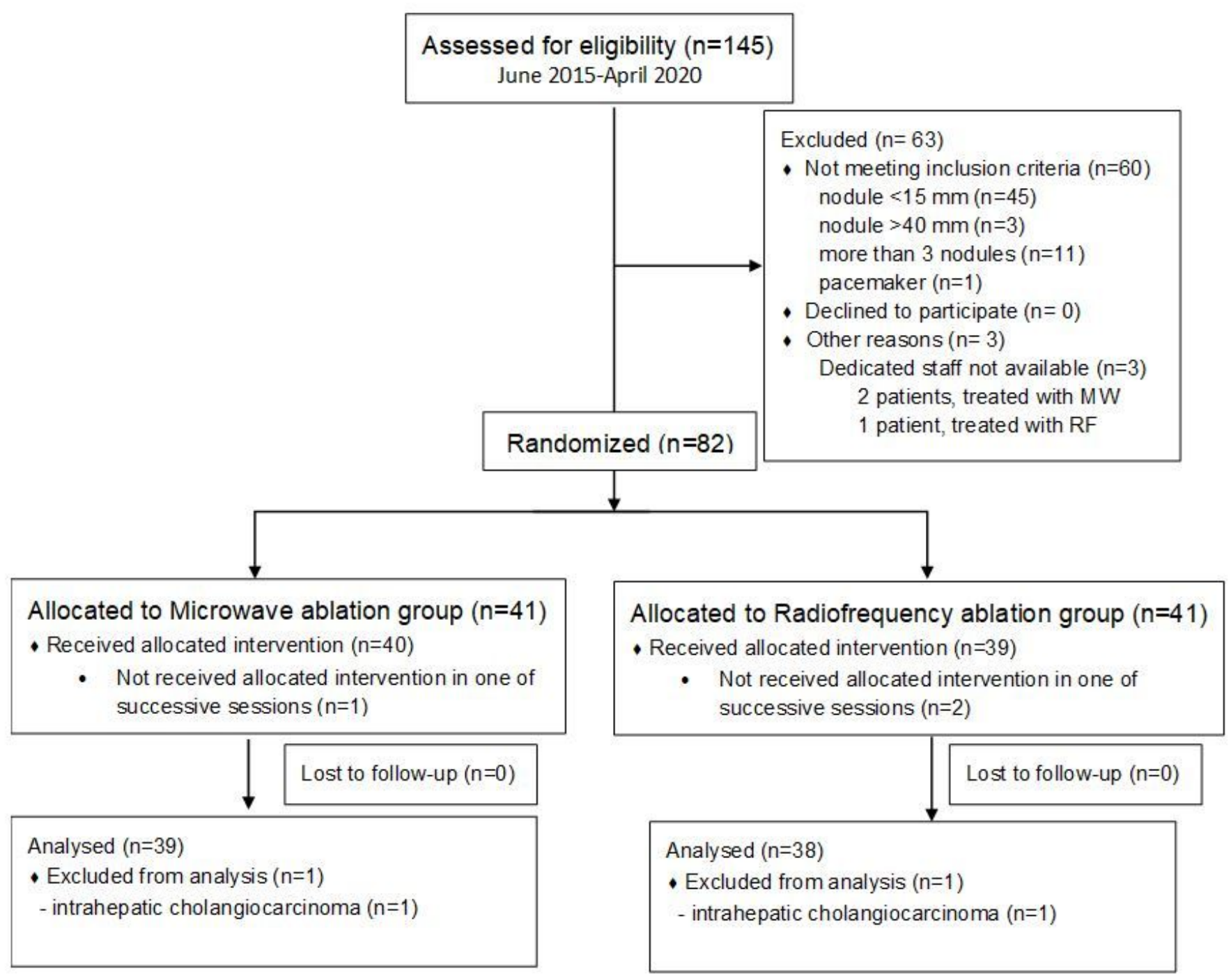

Figure 1

Clinical trial profile. 


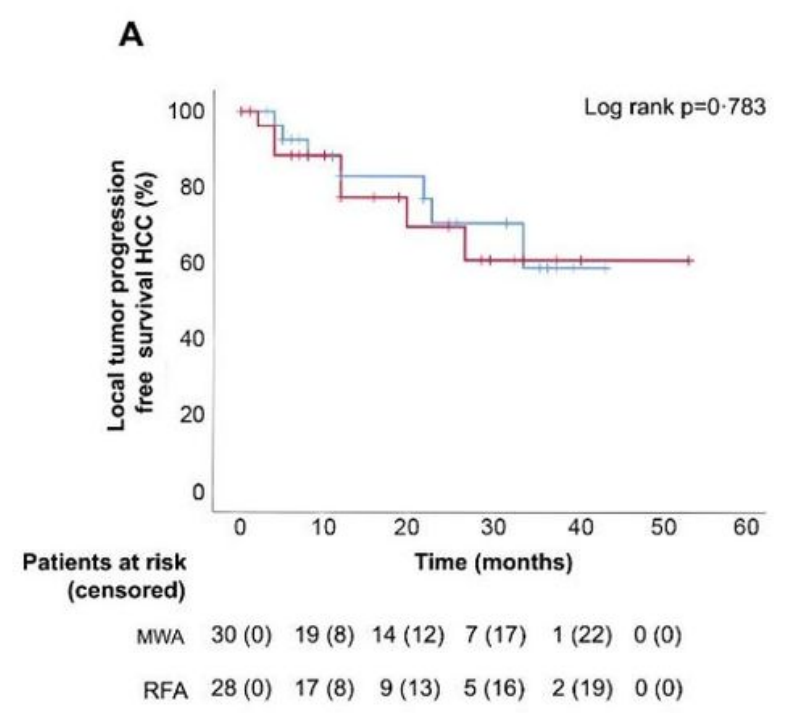

B

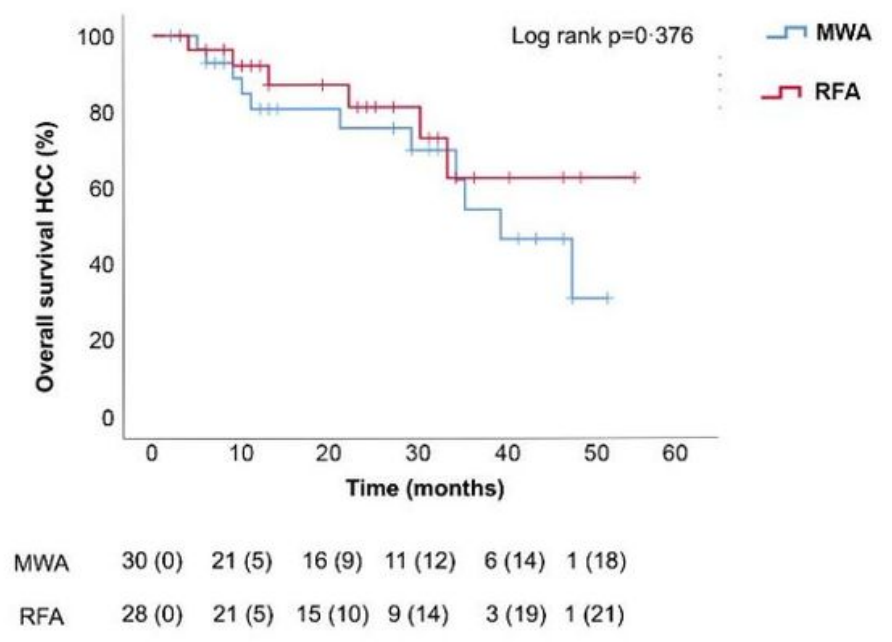

\section{Figure 2}

Kaplan-Meier curve of local tumor progression free survival $(A)$ and overall survival $(B)$ in hepatocarcinoma by group (MWA: microwave ablation; RFA: radiofrequency ablation).

\section{Supplementary Files}

This is a list of supplementary files associated with this preprint. Click to download.

- supplementaryfile.docx 